\title{
Progestin-primed ovarian stimulation vs mild stimulation in women with advanced age above 40: a retrospective cohort study
}

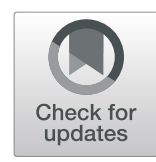

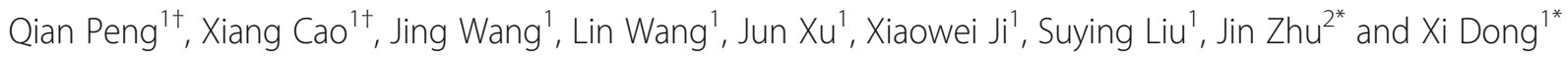

\begin{abstract}
Background: Previous studies have demonstrated that progestin-primed ovarian stimulation (PPOS) protocol was a feasible and efficient method in in vitro fertilization (IVF) cycle. However, its application in women with advanced age has not been determined yet. The purpose of this study was to investigate its efficacy in women aged $\geq 40$ years old.

Methods: This retrospective cohort study included patients with ages of $\geq 40$ years old at the time of ovarian stimulation. The embryonic and clinical outcome of mild stimulation and PPOS were compared. Primary outcome was topquality embryo rate on day 3, and secondary outcome was clinical pregnancy rate.

Results: Baseline characteristics of patients was similar in mild stimulation (122 cycles) and PPOS (47 cycles). No significant difference was found in the number of retrieved and mature oocytes and the fertilization and cleavage rates. Of interest, the rate of top-quality embryos was significantly higher in PPOS group $(50.08 \%$ vs 33.29\%, $p=$ $0.015)$, with an increasing trend of viable embryo rate (73.55\% vs $61.16 \%)$. A greater amount of gonadotropin was observed in PPOS group (2061.17 $\pm 1254.63 \mathrm{IU}$ vs $1518.14 \pm 547.25 \mathrm{IU}, p<0.05)$ in spite of comparable duration of stimulation. After FET cycle, no significant difference was found in the clinical pregnancy rates between mild stimulation (12.5\%) and PPOS group (16.7\%).

Conclusions: Higher percentage of top-quality embryos on Day 3 and comparable clinical pregnancy rate was obtained in PPOS protocol, which could be considered as a feasible ovarian stimulation protocol in women aged above 40 years old.
\end{abstract}

Keywords: Progestin-primed ovarian stimulation, Mild stimulation, Advanced maternal age, Embryo quality

\section{Background}

Since universal two-child policy took effect in 2015 in China, the number of women with advanced age over 40 undergoing IVF is rapidly growing. It is well known that oocyte yield and quality decrease with increased maternal age. Oocyte quality is the predominant determining factor for embryo quality, thus it affects the clinical pregnancy and live birth rates. According to the report from Society for Assisted Reproductive Technology (SART) in 2015 in the USA, live birth rate per egg retrieval cycle was only 11.1 and $3.6 \%$ for women aged

\footnotetext{
* Correspondence: zhujin88@hotmail.com; dong.xi@zs-hospital.sh.cn ${ }^{\dagger}$ Qian Peng and Xiang Cao are co-first authors.

${ }^{2}$ Department of Obstetrics and Gynecology, Shanghai Obstetrics and Gynecology Hospital, Fudan University, Shanghai, China

${ }^{1}$ Reproductive medicine centre, Zhongshan hospital, Fudan University, Shanghai, China
}

41-42 and $>42$ group, respectively. Cumulative live birth rate was slightly higher for $41-42$ group (12.6\%) and > 42 group (3.9\%) (https://www.sartcorsonline.com). Increased live birth rate and cumulative live birth rate were associated with increased number of retrieved oocytes [1, 2], which was affected by different ovarian stimulation protocols in IVF cycles. In the SART report system, there were four categories with different protocols including minimal stimulation, natural cycle, conventional stimulation and in vitro maturation. Unlike conventional stimulation protocol using high doses of gonadotropins, lower doses of gonadotropins were used in mild stimulation protocol based on the concept that more competent oocytes can be developed. Although mild stimulation protocol suffered from fewer numbers of oocytes or embryos obtained, it was proven to be safe 
and cost effectiveness. In addition, the cumulative pregnancy outcome was comparable to those that used conventional stimulation in normal and poor ovarian responders $[3,4]$.

PPOS protocol was initially employed by Prof. Kuang in 2015 [5]. The protocol has been demonstrated to result in good embryos and clinical outcomes in normal $[5,6]$ and poor ovarian responders $[7,8]$, and polycystic ovary syndrome (PCOS) patients [9]. The purposes of progesterone introduction into ovarian stimulation are to block luteinizing hormone (LH) surge. The LH surge is caused by the rising plasma estradiol and triggers ovulation, and a premature LH surge could lead to downregulation of oocyte yield. Medroxyprogesterone acetate (MPA) [5, 10], utrogestan [6] and dydrogesterone (DYG) [11] could be successfully served as an adjuvant to human menopausal gonadotropin (HMG) and effectively prevent premature LH surge. Gonadotropin-releasing hormone $(\mathrm{GnRH})$ antagonist could also be used, but about $38.3 \%$ of the patients suffered from premature luteinization [12]. In poor responders, the efficacy of PPOS protocol was only compared to natural cycle [8] and GnRH antagonist protocol [7], and progestin priming was proved to have no adverse or even better effect on clinical outcome. According to the Bologna criteria [13], women with advanced age $(\geq 40)$ is the most relevant risk factor for poor ovarian response. Therefore, the aim of the current retrospective cohort study was to compare the efficacy of PPOS and mild stimulation protocols in women with advanced age.

\section{Methods}

\section{Database}

This retrospective cohort study was conducted in the reproductive medicine centre of Zhongshan Hospital in Shanghai. Women with ages $\geq 40$ years undergoing IVF/ Intracytoplasmic sperm injection (ICSI) and subsequent frozen-thawed embryo transfer (FET) cycles between April 2016 and January 2019 were recruited. Written consents were obtained from all participants after counseling and IVF treatments. The patients were divided into two groups: mild stimulation group using clomiphene citrate (CC) with HMG and PPOS group. A proportion of them was followed by FET cycle.

\section{Ovarian stimulation protocol, embryo culture and vitrification}

The mild stimulation protocol was performed as followed: starting from Day 3 of menstrual cycle, patients received oral CC (Codal Synto Ltd., Cyprus) at $100 \mathrm{mg} / \mathrm{d}$ and HMG injection (Lizhu Pharmaceutical Trading Co., Zhuhai, China) at 150-225 IU/d daily. The patients in PPOS protocol received oral DYG (Duphaston; Abbott Biologicals B.V., Netherlands) at $20 \mathrm{mg} / \mathrm{d}$ and HMG injection at 150$225 \mathrm{IU} / \mathrm{d}$ daily from menstrual cycle Day 3 onwards. In both methods, the dosage of HMG was adjusted according to follicular diameter and estradiol level. The final oocyte maturation was triggered by intramuscular injection of human chorionic gonadotropin (hCG; Lizhu Pharmaceutical Trading Co.) at 5000-10000 IU or subcutaneously injection of triptorelin acetate $(200 \mu \mathrm{g})$ after evaluation of estradiol and progesterone levels in combination with the number of follicles with diameter $\geq 16 \mathrm{~mm}$.

Oocyte retrieval was performed under the guidance of transvaginal ultrasound. Follicles with $>10 \mathrm{~mm}$ in diameter were aspired at 34-36 h after ovulation trigger. Oocytes were fertilized by IVF/ICSI in G-IVF ${ }^{\mathrm{rm}}$ Fertilization media (Vitrolife, Göteborg, Sweden) with 10\% human serum albumin (HSA, Vitrolife). Embryos were cultured in either one step continuous single culture media (CSC; Irvine Scientific, CA, USA) or sequential culture media G- $1^{\mathrm{TM}} / \mathrm{G}-2^{\mathrm{TM}}$ medium (Vitrolife) with $10 \%$ HSA.

Embryo morphology was assessed and graded on Day 3 according to Cutting's criteria [14]. The embryos recorded as higher than $5 c(2 / 2)$ were considered as viable embryo, while those recorded as higher than 6c (3/4) were considered as top-quality embryo. Generally, two top-quality embryos were vitrified on Day 3, and supernumerary embryos were cultured continuously until blastocyst stage on Day 5 before vitrification. The vitrification procedure was performed following standard protocols using Irvine Scientific Freeze Kit (Irvine Scientific) with home-made straw carrier system.

\section{FET cycle, endometrial preparation and luteal support}

In some patients, hormonal treatment was used to prepare the endometrium for the FET cycles. Starting from menstrual cycle Day 3, patients received oral estradiol valerate (Progynova; Bayer, Germany) daily. From Day 10 onwards, serum hormone levels were measured, and endometrium growth were monitored by transvaginal ultrasound. When endometrial thickness $\geq 7 \mathrm{~mm}$ and progesterone $<3.18$ nmol/L were observed around Day 14, oral DYG (20 mg/ d) and vaginal progesterone $(600 \mathrm{mg} / \mathrm{d})$ were applied. Embryo transfer was scheduled on Day 3, 4 and 5. Luteal support was maintained until 8-10 weeks of gestation or negative $\beta$-hCG detection 2 weeks after transfer. Clinical pregnancy was defined as the presence of gestational sac determined by ultrasound around 7 weeks. FET cycles that included mixed embryos from different protocols were removed from this study.

\section{Data analysis}

The primary outcome was top-quality embryo rate on Day 3. The secondary outcome was clinical pregnancy rate. Statistical analysis was performed using SPSS (Version 22, SPSS Inc., Chicago, IL, USA). Continuous variables were presented as mean values and standard deviations and were compared using one-way ANOVA or Mann-Whitney test 
if applicable. Chi-square test and Fisher's exact test were used to compare percentages of qualitative variables and clinical outcomes. A logistic regression analysis was performed to assess for the associations between variables and pregnancy outcome. The data was shown as odds ratios (ORs) and 95\% confidence intervals (CIs).

\section{Results}

A total of 169 cycles including mild stimulation group (122 cycles) and PPOS group (47 cycles) were selected from our database during the study time period and were retrospectively examined. By the end of the research period (January 31, 2019), 74 FET cycles were completed, in which 56 cycles was from mild stimulation group and 18 cycles was from PPOS group. Demographic and basal characteristics of these patients are presented in Table 1 . No difference in age, body mass index (BMI), basal follicle stimulating hormone (FSH) and LH levels, factors and durations of infertility and percentages of primary infertility was found between the two groups. However, antral follicle count (AFC) was a bit lower $(7.09 \pm 3.29$ vs $8.23 \pm 3.17, p=0.039)$ while the number of previous IVF attempts was higher $(2.79 \pm 1.94$ vs $2.00 \pm 2.11, p=0.028$ ) in PPOS group when compared to mild stimulation group. Besides, basal estradiol $(135.06 \pm 84.75$ vs $192.91 \pm 149.42, p=0.006)$ and progesterone $(1.25 \pm 0.85$ vs $0.56 \pm 0.30, p<0.001)$ levels were significantly different between mild stimulation and PPOS groups.

The parameters and characteristics of the cycles in both groups were shown in Table 2. No obvious difference was

Table 1 Demographics and basal characteristics of patients in this study

\begin{tabular}{llll}
\hline Characteristics & Mild stimulation & PPOS & $p$ value \\
\hline Age & $43.32 \pm 2.49$ & $43.15 \pm 2.56$ & 0.692 \\
Basal FSH (mIU/ml) & $8.70 \pm 2.51$ & $7.91 \pm 2.21$ & 0.061 \\
Basal LH (mIU/ml) & $4.58 \pm 1.60$ & $4.68 \pm 1.98$ & 0.742 \\
Basal Estradiol (pmol/L) & $135.06 \pm 84.75$ & $192.91 \pm 149.42$ & 0.006 \\
Basal Progesterone (nmol/L) & $1.25 \pm 0.85$ & $0.56 \pm 0.30$ & $<0.001$ \\
BMI & $22.63 \pm 2.68$ & $21.95 \pm 2.63$ & 0.137 \\
AFC & $8.23 \pm 3.17$ & $7.09 \pm 3.29$ & 0.039 \\
Infertility factor (\%) & & & 0.500 \\
Female & $93(76.2 \%)$ & $36(76.7 \%)$ & $/$ \\
Male & $15(12.3 \%)$ & $3(6.4 \%)$ & $/$ \\
Both & $2(1.6 \%)$ & $2(4.3 \%)$ & $/$ \\
Unexplained & $12(9.8 \%)$ & $6(12.8 \%)$ & $/$ \\
Primary infertility (\%) & $21(17.2 \%)$ & $9(19.1 \%)$ & 0.944 \\
Infertility duration & $5.78 \pm 4.49$ & $6.33 \pm 5.95$ & 0.810 \\
Previous IVF attempts & $2.00 \pm 2.11$ & $2.79 \pm 1.94$ & 0.028 \\
\hline
\end{tabular}

Data was present as mean \pm SD observed in duration of ovarian stimulation, but the total amount of gonadotropin was significantly higher in PPOS group (2061.17 $\pm 1254.63, p<0.001)$. Moreover, LH, estradiol and progesterone levels on the trigger day were significantly lower in PPOS group (5.14 $\pm 2.81,5241.02 \pm$ 2712.04 and $1.83 \pm 2.57$, respectively, $p<0.05$ ). Meanwhile, the incidence of premature LH surge (defined as a $\mathrm{LH}$ level $\geq 10 \mathrm{IU} / \mathrm{L}$ and a progesterone level $\geq 3.18 \mathrm{nmol} / \mathrm{L}$ on trigger day [15]) was significantly lower in PPOS group. However, cancellation rate, number of retrieved and mature oocytes, fertilization and cleavage rates were comparable between mild stimulation and PPOS groups. The viable embryo rate was higher in PPOS group (73.55 \pm $36.58 \%$ vs $61.16 \pm 41.64 \%$ ), but the difference was not significant. However, top-quality embryo rate was significantly higher in PPOS group when compared with mild stimulation group $(50.08 \pm 41.65 \%$ vs $33.29 \pm 39.32 \%, p=$ $0.015)$. Linear regression analysis showed no significant association of top-quality embryo rate with progesterone level on the trigger day $(p=0.164)$.

In the subsequent FET cycle, clinical pregnancy rates were comparable between mild stimulation $(12.5 \%, 56$ cycles) and PPOS (16.7\%, 18 cycles) groups (Table 3). The number of transferred embryos at different stages and the endometrial thickness were not statistically different between mild stimulation and PPOS groups.

A binary logistic regression model was performed (Table 4). The dependent variable was clinical pregnancy outcome, and the independent variables included maternal age, BMI, duration of infertility, number of previous IVF attempts and transferred embryos per cycle, endometrial thickness and the ovarian stimulation type. Significant negative effect of maternal age $(\mathrm{OR}=0.644$, 95\% CI: 0.418-0.993) was found. There was a trend of adverse effect of duration of infertility $(\mathrm{OR}=0.848,95 \%$ CI:0.709-1.015), however, the result was not significant $(p=0.073)$. Furthermore, no effect was identified in other variables.

\section{Discussion}

The present study evaluated the efficacy of PPOS protocol in women with advanced age above 40 , when compared to mild stimulation using CC + HMG. The data suggested that PPOS protocol achieved better embryonic outcome, as demonstrated by the increased number of top-quality embryos obtained on day 3. Although statistically differences were observed in basal estradiol and progesterone between the groups, their levels were within normal range. AFC and previous IVF attempts were even worse in PPOS group.

The estradiol level was found higher while the total dose of gonadotropin was found lower on the trigger day in mild stimulation group. This may be due to the fact that, clomiphene citrate binds to the estrogen receptors on the 
Table 2 Characteristics of cycle parameters in both the group

\begin{tabular}{llll}
\hline Variables & Mild stimulation & PPOS & $p$ value \\
\hline Duration of ovarian stimulation (days) & $9.06 \pm 2.05$ & $8.49 \pm 2.03$ & 0.108 \\
Total dose of gonadotropin (IU) & $1518.14 \pm 547.25$ & $2061.17 \pm 1254.63$ & $<0.001$ \\
FSH on trigger day (mIU/ml) & $16.76 \pm 6.90$ & $16.51 \pm 4.80$ & 0.432 \\
LH on trigger day (mIU/ml) & $9.38 \pm 4.72$ & $5.14 \pm 2.81$ & $<0.001$ \\
Estradiol on trigger day (pmol/L) & $6959.59 \pm 4399.75$ & $5241.02 \pm 2712.04$ & 0.013 \\
Progesterone on trigger day (nmol/L) & $2.61 \pm 1.78$ & $1.83 \pm 2.57$ & 0.028 \\
Premature LH surge-no. (\%) & $15(12.3 \%)$ & $1(2.1 \%)$ & 0.044 \\
Cancelation rate (\%) & $31(25.4 \%)$ & $7(14.9 \%)$ & 0.207 \\
Number of oocytes retrieved & $3.57 \pm 2.77$ & $3.72 \pm 2.76$ & 0.740 \\
Number of mature oocytes & $3.08 \pm 2.39$ & $2.87 \pm 2.20$ & 0.602 \\
Fertilization rate (\%) & $79.05 \pm 31.50$ & $77.36 \pm 30.74$ & 0.754 \\
Cleavage rate (\%) & $87.25 \pm 30.25$ & $90.06 \pm 29.43$ & 0.585 \\
Viable embryo rate (\%) & $61.16 \pm 41.64$ & $73.55 \pm 36.58$ & 0.095 \\
Top-quality embryos rate (\%) & $33.29 \pm 39.32$ & $50.08 \pm 41.65$ & 0.015 \\
\hline
\end{tabular}

Data was present as mean \pm SD

hypothalamus and alters the negative feedback effect of estrogen, therefore induces the secretion of GnRH [16], which resulted in the higher level of estradiol in the mild stimulation group. In PPOS group, dydrogesterone application leads to pituitary suppression, which inhibits $\mathrm{GnRH}$ secretion [11]. Therefore, higher dosage of gonadotropin was required during ovarian stimulation.

Meanwhile, the progesterone level on the trigger day was higher in mild stimulation group as well. It has been demonstrated that estradiol levels on the day of hCG administration were significantly increased in women with progesterone elevation within $0.8-1.1 \mathrm{ng} / \mathrm{ml}$ [17]. The progesterone concentration in the present study was $2.61 \pm 1.78 \mathrm{nmol} / \mathrm{L}(0.82 \pm 0.56 \mathrm{ng} / \mathrm{ml})$ in mild stimulation group. The exact cause of progesterone elevation at the end of the follicular phase during ovarian stimulation of IVF is still obscure. It could be due to premature LH surges, which is caused by the actions of estradiol induced by gonadotrophins. Premature luteinization exists in up to $15-20 \%$ of IVF cycles, which could lead to cancellation of cycles [18]. Consistently, the percentage of premature LH surge was significantly higher in mild stimulation when compared with PPOS group. There was also a trend of higher cancelation rate, though it was not statistically significant. It is well-known that IVF pregnancy outcome decreased dramatically in women with advanced age. They suffered from higher risk of premature luteinization, which could be due to changes of gene expressions related to gonadotropin activity. Previous study reported the down-regulation of FSH receptor (FSHR) but up-regulated LH receptor (LHCGR) and progesterone receptor (PGR) gene expressions in granulosa cells in women aged more than 43 [19]. The increased expression of LHCGR and PGR and decreased FSHR has been reported in luteinized granulosa cells [20-22]. Progesterone has been reported to play an important role in oocyte nuclear and cytoplasmic maturation and developmental competence [23-25]. The better embryonic outcome with progestin administration in PPOS protocol may be due to modulated interaction between progesterone and its receptor in aged female. However, the impact of endogenous progesterone level

Table 3 Clinical outcomes of FET cycles

\begin{tabular}{|c|c|c|c|}
\hline Variables & Mild stimulation & PPOS & $p$ value \\
\hline Number of thawed cycles & 56 & 18 & \\
\hline Number of transferred embryos at different stage & & & 0.134 \\
\hline Number of Cleavage-stage embryo transferred-no. (\%) & $34(60.7 \%)$ & $11(61.1 \%)$ & / \\
\hline Number of Blastocyst transferred-no. (\%) & $18(32.1 \%)$ & $3(16.7 \%)$ & / \\
\hline Number of Cleavage-stage and Blastocyst embryo transferred-no. (\%) & $4(7.1 \%)$ & $4(22.2 \%)$ & / \\
\hline Endometrial thickness (mm) & $9.78 \pm 1.93$ & $9.00 \pm 1.97$ & 0.140 \\
\hline Clinical Pregnancy rate - no. (\%) & $7(12.5 \%)$ & $3(16.7 \%)$ & 0.697 \\
\hline
\end{tabular}


Table 4 Logistic regression analysis of clinical pregnancy outcome in the study

\begin{tabular}{lll}
\hline Independent variables & OR [95\% Cl] & $p$ value \\
\hline Maternal age & $0.644[0.418,0.993]$ & 0.046 \\
BMI & $0.946[0.662,1.352]$ & 0.761 \\
Duration of infertility & $0.848[0.709,1.015]$ & 0.073 \\
Number of previous IVF attempts & $0.924[0.631,1.354]$ & 0.685 \\
Number of transferred embryos per cycle & $0.975[0.238,3.996]$ & 0.971 \\
Endometrial thickness & $1.289[0.851,1.954]$ & 0.231 \\
Mild stimulation vs PPOS & $0.544[0.137,2.159]$ & 0.386 \\
\hline
\end{tabular}

on the trigger day on embryo quality is still controversial. A large systematic review and meta-analysis of over 60 , 000 cycles study has demonstrated that the detrimental effect of progesterone concentration on clinical pregnancy started from range of $0.8-1.1 \mathrm{ng} / \mathrm{ml}$. However, such effect was observed only in women undergoing fresh IVF cycles but not FET cycles, because it was hypothesized that it was through its action on the endometrium [17]. In the current study, FET cycles were used and the progesterone level in the mild stimulation group did not exceed the marginal value reported in that study. Another report showed that premature progesterone elevation was not associated with embryo quality [26]. In agreement with that study, our result also showed no association between the progesterone level with top-quality rate. There was one report suggesting a negative association between these two parameters [27]. However, the cutoff value of progesterone detrimental to embryo quality was $2.0 \mathrm{ng} / \mathrm{ml}$ in the study, which was much higher than that in the mild stimulation group in our study. Taken together, lower LH level, blockage of premature LH surge and better embryonic outcome indicated that PPOS could be served as a feasible ovarian stimulation method for aged women.

The first randomized cohort study of PPOS was reported by Prof. Kuang in 2015 with the combination of MPA and HMG (150-225 IU/d) administration from menstruation day 3 onwards [5]. Although the duration of stimulation was significantly longer and total dose of HMG was significantly higher in PPOS when compared to conventional short protocol in normal responders, no difference was found in the number of mature oocytes and frozen embryos between the two groups [5]. There was also no difference found in the implantation rate, clinical pregnancy rate, miscarriage rate and live birth rate after FET cycles. However, a recent retrospective study has shown significantly higher matured oocytes rate, fertilization rate, good-quality embryo rate, clinical pregnancy and live birth rates in PPOS protocol when compared to GnRH antagonist protocol in poor responders [28]. The good-quality embryo rates in their PPOS group was $70 \%$ [28], which was higher than that of $50 \%$ in our study. This could be due to more stringent criteria employed during our embryo morphology assessment on day 3 . The good-quality embryos in their study referred to those with at least six blastomeres and fragments $<50 \%$ [28], while ours were defined as embryos with at least six blastomeres and fragments $<20 \%$. Meanwhile, same PPOS protocol was compared to natural cycle in poor responders with diminished ovarian reserve in a non-randomized prospective cohort study [8]. Their result showed that half of the patients in natural cycle group had LH surges while none was observed in PPOS group, and the number of mature oocytes and viable embryos was significantly higher in PPOS group. The proportion of cycles with at least one viable embryo in their PPOS group was $50.0 \%$, while the viable embryo rate in our study was $73 \%$. Unlike the protocols in our study, they used exogenous progesterone and low dose of HMG in the late follicular phase, and the result showed that progestin primed minimal stimulation protocol could also efficiently control the development of dominant follicle and embryo quality in poor responders [8]. In patients with high BMI, higher implantation rate, clinical pregnancy rate and live birth rate were associated with PPOS protocol when compared with conventional short protocol [29]. The efficacy of PPOS was also compared to short agonist protocol in a pilot study of 60 PCOS patients [9]. In contradiction to previous result in natural cycle [8], no difference in the number of oocytes collected and ongoing pregnancy rates but a higher total dose of HMG was found in the PPOS group of PCOS patients. Therefore, the controversy still exists due to different outcomes in different populations. DYG was used as exogeneous progestin in this study. Even though greater assumption of gonadotropin was observed in PPOS group, it is cost-effective as the price of HMG is comparatively low. DYG is an alternative to MPA in PPOS protocol in a recent prospective randomized controlled trial (RCT) [11]. The study showed that no significant difference was found between DYG and MPA in terms of number of oocytes retrieved, the viable embryo rate per oocyte and the clinical pregnancy rate.

The use of progesterone to block the LH surge has been summarized in a recent review [30]. Either endogenous with luteal phase stimulation and exogenous with PPOS was confirmed effective. Several studies have demonstrated that high progesterone in follicular and luteal phase stimulation had no adverse effect on oocyte and embryo qualities. Retrospective study demonstrated that endogenous progesterone alone was sufficient to block the LH surge and agonist or antagonist was unnecessary. Implantation rates were similar in luteal stimulation and follicular stimulation [31, 32]. Multiple stimulation protocols have been derived from ovarian stimulation protocol 
under high progesterone, such as 'duostim' [33] or 'Shanghai protocol' [34]. It has an advantage for fertility perseveration in the urgent context of oncology. However, the application and cost-effectiveness of the stimulation protocol for poor responders was still undetermined due to retrospective studies with few patients. Our study also suffered from small sample size and its nature of retrospective design. Future RCT study is required. Besides, the primary outcome was top-quality embryos on day 3 , which may be not the best parameter to predict the prognosis of patients undergoing IVF cycle. The current study would provide an alternative approach for patients with advanced ages in obtaining embryos with better quality, which potentially alleviate their stress during IVF treatment cycles.

\section{Conclusions}

In conclusion, our data showed that the use of progesterone during ovarian stimulation could efficiently block the LH surge in women with advanced age, and it did not affect the number of oocytes collected but resulted in better embryonic outcome on day 3 . Such protocol had a promising application in aged patients who wished to reserve embryos in a short period and shorten their time to pregnancy.

\section{Abbreviations \\ AFC: Antral follicle counting; BMI: Body mass index; CC: Clomiphene citrate; Cls: Confidence intervals; DYG: Dydrogesterone; FET: Frozen-thawed embryo transfer; FSH: Follicle stimulating hormone; FSHR: FSH receptor; GnRH: Gonadotropin-releasing hormone; hCG: Human chorionic gonadotropin; HMG: Human menopausal gonadotropin; HSA: Human serum albumin; ICSI: Intracytoplasmic sperm injection; IVF: In vitro fertilization; LH: Luteinizing hormone; LHCGR: LH receptor; MPA: Medroxyprogesterone acetate; ORs: Odds ratios; PCOS: Polycystic ovary syndrome; PGR: Progesterone receptor; PPOS: Progestin-primed ovarian stimulation; SART: Society for Assisted Reproductive Technology}

\section{Acknowledgements}

Not applicable

\section{Authors' contributions}

PQ conceptualized and wrote the manuscript. PQ and CX performed data collection and analysis. WJ, WL, XJ, JXW, LSY reviewed manuscript. ZJ and DX were responsible for overall supervision. All authors contributed to the interpretation of the data and approved the final submission of the manuscript.

\section{Funding}

This work is partly funded by Zhongshan Hospital Youth Fund (2018ZSQN42 to Peng Qian).

\section{Availability of data and materials}

The datasets used and/or analyzed during the current study are available from the corresponding author on reasonable request.

\section{Ethics approval and consent to participate}

Not applicable

\section{Consent for publication}

Not applicable

\section{Competing interests}

The authors declare that they have no competing interests.

Received: 7 May 2019 Accepted: 27 August 2019

Published online: 09 November 2019

\section{References}

1. Drakopoulos P, Blockeel C, Stoop D, Camus M, de Vos M, Tournaye H, Polyzos NP. Conventional ovarian stimulation and single embryo transfer for IVF/ICSI. How many oocytes do we need to maximize cumulative live birth rates after utilization of all fresh and frozen embryos? Hum Reprod. 2016;31:370-6.

2. Baker VL, Brown MB, Luke B, Conrad KP. Association of number of retrieved oocytes with live birth rate and birth weight: an analysis of 231,815 cycles of in vitro fertilization. Fertil Steril. 2015;103:931-8 e932.

3. Nargund G, Datta AK, Fauser B. Mild stimulation for in vitro fertilization. Fertil Steril. 2017;108:558-67.

4. Youssef MA, van Wely M, Al-Inany $H$, Madani T, Jahangiri $\mathrm{N}$, Khodabakhshi $\mathrm{S}$, Alhalabi M, Akhondi M, Ansaripour S, Tokhmechy R, et al. A mild ovarian stimulation strategy in women with poor ovarian reserve undergoing IVF: a multicenter randomized non-inferiority trial. Hum Reprod. 2017;32:112-8.

5. Kuang Y, Chen Q, Fu Y, Wang Y, Hong Q, Lyu Q, Ai A, Shoham Z. Medroxyprogesterone acetate is an effective oral alternative for preventing premature luteinizing hormone surges in women undergoing controlled ovarian hyperstimulation for in vitro fertilization. Fertil Steril. 2015;104:62-70 e63.

6. Zhu X, Zhang X, Fu Y. Utrogestan as an effective oral alternative for preventing premature luteinizing hormone surges in women undergoing controlled ovarian hyperstimulation for in vitro fertilization. Medicine (Baltimore). 2015;94:e909.

7. Huang P, Tang M, Qin A. Progestin-primed ovarian stimulation is a feasible method for poor ovarian responders undergoing in IVF/ICSI compared to a GnRH antagonist protocol: a retrospective study. J Gynecol Obstet Hum Reprod. 2019;48:99-102.

8. Chen Q, Wang Y, Sun L, Zhang S, Chai W, Hong Q, Long H, Wang L, Lyu Q, Kuang $Y$. Controlled ovulation of the dominant follicle using progestin in minimal stimulation in poor responders. Reprod Biol Endocrinol. 2017;15:71.

9. Wang Y, Chen Q, Wang N, Chen H, Lyu Q, Kuang Y. Controlled ovarian stimulation using medroxyprogesterone acetate and hMG in patients with polycystic ovary syndrome treated for IVF: a double-blind randomized crossover clinical trial. Medicine (Baltimore). 2016;95:e2939.

10. Dong J, Wang Y, Chai WR, Hong QQ, Wang NL, Sun LH, Long H, Wang L, Tian H, Lyu QF, et al. The pregnancy outcome of progestin-primed ovarian stimulation using 4 versus $10 \mathrm{mg}$ of medroxyprogesterone acetate per day in infertile women undergoing in vitro fertilisation: a randomised controlled trial. BJOG. 2017:124:1048-55.

11. Yu S, Long H, Chang HY, Liu Y, Gao H, Zhu J, Quan X, Lyu Q, Kuang Y, Ai A. New application of dydrogesterone as a part of a progestin-primed ovarian stimulation protocol for IVF: a randomized controlled trial including 516 first IVF/ICSI cycles. Hum Reprod. 2018;33:229-37.

12. Bosch E, Valencia I, Escudero E, Crespo J, Simon C, Remohi J, Pellicer A. Premature luteinization during gonadotropin-releasing hormone antagonist cycles and its relationship with in vitro fertilization outcome. Fertil Steril. 2003;80:1444-9.

13. Ferraretti AP, La Marca A, Fauser BC, Tarlatzis B, Nargund G, Gianaroli L, Definition EPOR. ESHRE consensus on the definition of 'poor response' to ovarian stimulation for in vitro fertilization: the Bologna criteria. Hum Reprod. 2011;26:1616-24.

14. Cutting R, Morroll D, Roberts SA, Pickering S, Rutherford A, BFS, ACE. Elective single embryo transfer: guidelines for practice British fertility society and association of clinical embryologists. Hum Fertil (Camb). 2008:11:131-46.

15. Lambalk CB, Leader A, Olivennes F, Fluker MR, Andersen AN, Ingerslev J, Khalaf Y, Avril C, Belaisch-Allart J, Roulier R, Mannaerts B. Treatment with the $\mathrm{GnRH}$ antagonist ganirelix prevents premature LH rises and luteinization in stimulated intrauterine insemination: results of a double-blind, placebocontrolled, multicentre trial. Hum Reprod. 2006;21:632-9.

16. Kato K, Ezoe K, Yabuuchi A, Fukuda J, Kuroda T, Ueno S, Fujita H, Kobayashi T. Comparison of pregnancy outcomes following fresh and electively frozen single blastocyst transfer in natural cycle and clomiphene-stimulated IVF cycles. Hum Reprod Open. 2018;2018:hoy006.

17. Venetis CA, Kolibianakis EM, Bosdou JK, Tarlatzis BC. Progesterone elevation and probability of pregnancy after IVF: a systematic review and metaanalysis of over 60000 cycles. Hum Reprod Update. 2013;19:433-57. 
18. de Ziegler D, Andersen CY, Stanczyk FZ, Ayoubi JM. Endocrine mechanisms and assay issues in premature progesterone elevation in assisted reproductive technology. Fertil Steril. 2018;109:571-6.

19. Wu YG, Barad DH, Kushnir VA, Lazzaroni E, Wang Q, Albertini DF, Gleicher N. Aging-related premature luteinization of granulosa cells is avoided by early oocyte retrieval. J Endocrinol. 2015;226:167-80

20. Natraj U, Richards JS. Hormonal regulation, localization, and functional activity of the progesterone receptor in granulosa cells of rat preovulatory follicles. Endocrinology. 1993;133:761-9.

21. Luborsky JL, Thiruppathi P, Rivnay B, Roussev R, Coulam C, Radwanska E. Evidence for different aetiologies of low estradiol response to FSH: agerelated accelerated luteinization of follicles or presence of ovarian autoantibodies. Hum Reprod. 2002;17:2641-9.

22. Jeppesen JV, Kristensen SG, Nielsen ME, Humaidan P, Dal Canto M, Fadini R, Schmidt KT, Ernst E, Yding Andersen C. LH-receptor gene expression in human granulosa and cumulus cells from antral and preovulatory follicles. J Clin Endocrinol Metab. 2012;97:E1524-31.

23. Fair T, Lonergan P. The role of progesterone in oocyte acquisition of developmental competence. Reprod Domest Anim. 2012;47(Suppl 4):142-7.

24. Aparicio IM, Garcia-Herreros M, O'Shea LC, Hensey C, Lonergan P, Fair T. Expression, regulation, and function of progesterone receptors in bovine cumulus oocyte complexes during in vitro maturation. Biol Reprod. 2011;84: 910-21.

25. Friberg PA, Larsson DG, Billig $\mathrm{H}$. Transcriptional effects of progesterone receptor antagonist in rat granulosa cells. Mol Cell Endocrinol. 2010;315: 121-30.

26. Hill MJ, GDt R, Healy MW, Richter KS, Levy G, AH DC, Levens ED, Suthar G, Widra E, Levy MJ. Are good patient and embryo characteristics protective against the negative effect of elevated progesterone level on the day of oocyte maturation? Fertil Steril. 2015;103:1477-84 e1471-1475.

27. Huang $B$, Ren $X, W u L$, Zhu L, Xu B, Li Y, Ai J, Jin L. Elevated progesterone levels on the day of oocyte maturation may affect top quality embryo IVF cycles. PLoS One. 2016;11:e0145895.

28. Huang $P$, Tang M, Qin A. Progestin-primed ovarian stimulation is a feasible method for poor ovarian responders undergoing in IVF/ICSI compared to a GnRH antagonist protocol: a retrospective study. J Gynecol Obstet, Hum Reprod. 2018

29. Wang L, Yin M, Liu Y, Chen Q, Wang Y, Ai A, Fu Y, Yan Z, Jin W, Long H, et al. Effect of frozen embryo transfer and progestin-primed ovary stimulation on IVF outcomes in women with high body mass index. Sci Rep. 2017;7:7447.

30. Massin N. New stimulation regimens: endogenous and exogenous progesterone use to block the $\mathrm{LH}$ surge during ovarian stimulation for IVF. Hum Reprod Update. 2017;23:211-20.

31. Wang N, Wang Y, Chen Q, Dong J, Tian H, Fu Y, Ai A, Lyu Q, Kuang Y. Luteal-phase ovarian stimulation vs conventional ovarian stimulation in patients with normal ovarian reserve treated for IVF: a large retrospective cohort study. Clin Endocrinol. 2016;84:720-8.

32. Li Y, Yang W, Chen X, Li L, Zhang Q, Yang D. Comparison between follicular stimulation and luteal stimulation protocols with clomiphene and HMG in women with poor ovarian response. Gynecol Endocrinol. 2016;32:74-7.

33. Ubaldi FM, Capalbo A, Vaiarelli A, Cimadomo D, Colamaria S, Alviggi C, Trabucco E, Venturella R, Vajta G, Rienzi L. Follicular versus luteal phase ovarian stimulation during the same menstrual cycle (DuoStim) in a reduced ovarian reserve population results in a similar euploid blastocyst formation rate: new insight in ovarian reserve exploitation. Fertil Steril. 2016; 105:1488-95 e1481.

34. Kuang Y, Chen Q, Hong Q, Lyu Q, Ai A, Fu Y, Shoham Z. Double stimulations during the follicular and luteal phases of poor responders in IVF/ICSI programmes (Shanghai protocol). Reprod BioMed Online. 2014;29: $684-91$

\section{Publisher's Note}

Springer Nature remains neutral with regard to jurisdictional claims in published maps and institutional affiliations.

Ready to submit your research? Choose BMC and benefit from:

- fast, convenient online submission

- thorough peer review by experienced researchers in your field

- rapid publication on acceptance

- support for research data, including large and complex data types

- gold Open Access which fosters wider collaboration and increased citations

- maximum visibility for your research: over $100 \mathrm{M}$ website views per year

At BMC, research is always in progress.

Learn more biomedcentral.com/submissions 